\title{
Attenuation of myocardial fibrosis with curcumin is mediated by modulating expression of angiotensin II ATI/AT2 receptors and ACE2 in rats
}

This article was published in the following Dove Press journal:

Drug Design, Development and Therapy

II November 2015

Number of times this article has been viewed

\author{
Xue-Fen Pang' \\ Li-Hui Zhang ${ }^{2}$ \\ Feng Bai' \\ Ning-Ping Wang ${ }^{3}$ \\ Ron E Garner ${ }^{3}$ \\ Robert J McKallip ${ }^{4}$ \\ Zhi-Qing Zhao',3 \\ 'Department of Physiology, \\ Shanxi Medical University, Taiyuan, \\ Shanxi, People's Republic of China; \\ ${ }^{2}$ Department of Cardiology, Shanxi \\ Academy of Medical Sciences and \\ Shanxi Dayi Hospital, Shanxi Medical \\ University, Taiyuan, Shanxi, People's \\ Republic of China; ${ }^{3}$ Department of \\ Basic Biomedical Sciences, Mercer \\ University School of Medicine, \\ Savannah, GA, USA; ${ }^{4}$ Division of \\ Basic Biomedical Sciences, Mercer \\ University School of Medicine, \\ Macon, GA, USA
}

\begin{abstract}
Curcumin is known to improve cardiac function by balancing degradation and synthesis of collagens after myocardial infarction. This study tested the hypothesis that inhibition of myocardial fibrosis by curcumin is associated with modulating expression of angiotensin II (Ang II) receptors and angiotensin-converting enzyme 2 (ACE2). Male Sprague Dawley rats were subjected to Ang II infusion (500 ng/ $\mathrm{kg} / \mathrm{min}$ ) using osmotic minipumps for 2 and 4 weeks, respectively, and curcumin (150 mg/kg/day) was fed by gastric gavage during Ang II infusion. Compared to the animals with Ang II infusion, curcumin significantly decreased the mean arterial blood pressure during the course of the observation. The protein level of the Ang II type 1 (AT1) receptor was reduced, and the Ang II type 2 (AT2) receptor was up-regulated, evidenced by an increased ratio of the AT2 receptor over the AT1 receptor in the curcumin group $(1.2 \pm 0.02 \%)$ vs in the Ang II group $(0.7 \pm 0.03 \%, P<0.05)$. These changes were coincident with less locally expressed AT1 receptor and enhanced AT2 receptor in the intracardiac vessels and intermyocardium. Along with these modulations, curcumin significantly decreased the populations of macrophages and alpha smooth muscle actin-expressing myofibroblasts, which were accompanied by reduced expression of transforming growth factor beta 1 and phosphorylated-Smad2/3. Collagen I synthesis was inhibited, and tissue fibrosis was attenuated, as demonstrated by less extensive collagen-rich fibrosis. Furthermore, curcumin increased protein level of ACE2 and enhanced its expression in the intermyocardium relative to the Ang II group. These results suggest that curcumin could be considered as an add-on therapeutic agent in the treatment of fibrosis-derived heart failure patient who is intolerant of ACE inhibitor therapy.
\end{abstract}

Keywords: angiotensin II receptors, angiotensin-converting enzyme 2, curcumin, collagen, myocardial fibrosis

\section{Introduction}

Myocardial fibrosis can occur as a result of hypertension, ischemic injury, or valvular heart disease, and is a major cause of left ventricular diastolic dysfunction that accounts for $40 \%$ to $50 \%$ of heart failure patients. ${ }^{1}$ Pathologically, fibrosis is characterized by the accumulation of fibrillar collagens, and myocardia fibrosis develops from the perivascular area to the intermyocardium with a diffuse distribution pattern. The lack of resolution of this excessive collagen deposition in tissue is associated with reduction of cardiac muscle compliance, filling impairment, and ultimately heart failure., ${ }^{2,3}$

Pharmacological interventions, aimed at inhibiting conversion of angiotensin (Ang) I to Ang II with angiotensin-converting enzyme (ACE) inhibitors or a blockade of Ang II type 1 (AT1) receptor by AT1 receptor antagonists, have defined the role of systemic and locally generated Ang II within myocardium in the development of fibrosis-derived heart failure. In response to Ang II stimulation, activation of the AT1
Correspondence: Zhi-Qing Zhao Department of Basic Biomedical Sciences, Mercer University School of Medicine, 4700 Water Avenue, Savannah, GA 3I404, USA

Email zhao_z@mercer.edu 
receptor induces inflammatory response, vascular constriction, interstitial collagen deposition, and tissue fibrosis. However, up-regulation of the Ang II type 2 (AT2) receptor may evoke cardioprotective effects by countervailing over-all deleterious effects derived by stimulating the AT1 receptor. ${ }^{4}$

Angiotensin-converting enzyme 2 (ACE2) is a homologue of ACE, sharing $42 \%$ sequence identity and $61 \%$ sequence similarity with the catalytic domain of ACE, but is not inhibited by ACE inhibitors. An increase in ACE2 activity is associated with a decrease in tissue level of Ang II through degradation of Ang II to Ang-(1-7), hence functioning effectively as a negative regulator of Ang II-mediated vasoconstriction, proliferation, and fibrosis. ${ }^{5}$ Attenuation of ACE2 activity is deleterious to the heart, because it leads to cardiac hypertrophy and cardiac dysfunction, due in part to increased Ang II stimulation of the AT1 receptor. In ACE2deficient hearts, blockade of the AT1 receptor has been shown to reduce the susceptibility to Ang II-potentiated heart failure, suggesting that down-regulation of ACE2 expression is mediated via AT1 receptor activation. ${ }^{6}$

Currently, the ACE inhibitor or the AT1 receptor antagonist has become one of the most successful therapeutic approaches in patients with hypertension and heart failure. However, data from clinical observations have also revealed that the use of ACE inhibitors is associated with a higher rate of dry cough and angioedema, while hypotensive symptoms are often identified with the use of AT1 receptor antagonists. $^{7}$ Therefore, adjunctive therapies to avoid these unfavorable effects and to reduce morbidity and mortality of cardiovascular diseases by inhibiting Ang II activation, remain an active area of investigation.

Curcumin, the yellow pigment extracted from the rhizomes of the plant Curcuma longa, exhibits diverse pharmacologic properties, such as anti-oxidant, anti-inflammation, and antifibrosis properties. ${ }^{8,9}$ We have previously demonstrated that dietary administration of curcumin reduces infarct size and improves cardiac function in the rat model of infarctionelicited heart failure. ${ }^{10}$ However, we do not know whether curcumin has a direct effect on Ang II-induced myocardial fibrosis. In the present study, we tested the hypothesis that curcumin inhibits the myocardial fibrotic process through altering expression of Ang II AT1/AT2 receptors and ACE2. Our results revealed AT1 receptor antagonism, activation of the AT2 receptor, and ACE2 up-regulation by curcumin. Moreover, these curcumin-dependent actions inhibited myocardial fibrosis by attenuating accumulation of macrophages/myofibroblasts and by activating transforming growth factor beta 1 (TGF $\beta 1$ )/Smads signaling pathways.

\section{Materials and methods Ethical approval}

All animals received human care in compliance with The Guide for the Care of Use of Laboratory Animals, published by the US National Institutes of Health $(\mathrm{NIH}$; 8th edition revised, 2011). ${ }^{10}$ The procedures in this study were approved by the Institutional Animal Care and Use Committee, Mercer University School of Medicine, Savannah, GA, USA.

\section{In vivo rat model of angiotensin II infusion with an osmotic minipump}

Male Sprague Dawley rats weighing 200 \pm 10 g (Harlan Laboratories, Indianapolis, IN, USA) were used in the present study. An intraperitoneal injection of a ketamine $(90 \mathrm{mg} / \mathrm{kg})$ and xylazine $(10 \mathrm{mg} / \mathrm{kg})$ mixture was selected to anesthetize the rats on the day before a minipump implantation. The skin was swabbed with povidone-iodine and alcohol after shaving. An incision between the scapulae was made, and a small pocket was produced using a hemostat to spread the subcutaneous connective tissue. An osmotic minipump was inserted into the pocket and the incision was closed with sutures. The osmotic minipumps (models 2002 and 2004; DURECT Corp, Cupertino, CA, USA) were used to perfuse Ang II (purchased from Sigma-Aldrich Co, St Louis, MO, USA). These pumps were pre-incubated in the tubes with $0.9 \%$ sterile saline only or saline containing Ang II at $37^{\circ} \mathrm{C}$ overnight prior to implantation.

\section{Blood pressure measurement and study groups}

A non-invasive blood pressure (NIBP) measuring system was selected to detect the NIBP change from all animals using a pulse transducer (PowerLab ML125 NIBP Controller, ADInstruments, Colorado Springs, CO, USA). This system operates by occluding blood flow to the tail with a specialized cuff. The transducer intermittently measures blood pressure based on the periodic occlusion of blood flow in the tail. The measurement of NIBP was considered valid only when three consecutive readings did not differ by more than $10 \mathrm{mmHg}$.

Two observational points (ie, 2 and 4 weeks of Ang II infusion) were selected for all experimental groups. Animals were randomized into one of three groups ( $n=6$ / each observational period): 1) the control group, in which rats were received Ang II infusion only at a rate of $500 \mathrm{ng} / \mathrm{kg} / \mathrm{min}$; 2) the Ang II plus curcumin group, in which curcumin (Sigma-Aldrich Co) was administered by gastric gavage at a dose of $150 \mathrm{mg} / \mathrm{kg} /$ day during Ang II 
infusion; ${ }^{10}$ and 3 ) the sham plus curcumin (normal) group, in which rats were infused with a saline pump and curcumin was given by gastric gavage at the same dose as in the curcumin group.

\section{Tissue preparation for histological examination}

At the end of the experimental period in each group, the rat was euthanized, and the heart was removed. Transversal myocardial slices were fixed in 10\% phosphate-buffered formalin solution for 24 hours, and were then embedded in paraffin wax for histological analysis, as we have reported previously. ${ }^{10}$ Tissue sections ( $6 \mu \mathrm{m}$ thick) were made using a microtome (Leica RM2135, Meyer Instruments, Inc, Houston, TX, USA). For Western blotting analysis, small pieces of transmural tissue sections from the left ventricle were frozen immediately in tubes using liquid nitrogen and kept at $-80^{\circ} \mathrm{C}$ until use.

\section{Protein levels of Ang II receptors, ACE2, TGF $\beta$ - I, Smads, and collagens}

The freshly frozen transmural tissue samples were homogenized in lysis buffer, and protein concentration was measured by the Bio-Rad detergent compatible protein assay (Bio-Rad Laboratories Inc., Hercules, CA, USA), as we have previously reported. ${ }^{10}$ Briefly, the protein was boiled and loaded onto gradient sodium dodecyl sulfate (SDS)-polyacrylamide gel using Mini-Protean ${ }^{\circledR}$ II Dual Stab Cell (Bio-Rad). Electrophoretically separated proteins were transferred from polyacrylamide to nitrocellulose membranes, and were subsequently probed with the following antibodies: 1) rabbit anti-AT1 and AT2 receptor polyclonal antibodies; 2) a rabbit anti-ACE2 polyclonal antibody (Santa Cruz Biotechnology Inc, Dallas, TX, USA); 3) a mouse antiTGF $\beta 1$ monoclonal antibody (Abcam plc, Cambridge, MA, USA); 4) mouse anti-collagen type I and III monoclonal antibodies (Sigma-Aldrich Co); and 5) rabbit anti-phosphoSmad2/3 monoclonal antibodies (Cell Signaling Technology, Danvers, MA, USA). Bound antibody was detected with the species-appropriate horseradish peroxidase-conjugated anti-immunoglobulin $\mathrm{G}$ ( $\mathrm{IgG})$. The membrane was then incubated with chemiluminescence detection reagents, and binding was detected by X-ray film exposure. Actin was used as a protein-loading standard control to normalize the bands. The scanned images were imported into the ImageJ software (NIH, Bethesda, MD, USA). The final results were calculated as a ratio of intensity from each band divided by actin.
Detection of ATI/AT2 receptors, ACE2, macrophages, and alpha smooth muscle actin-expressing myofibroblasts

Immunohistochemical staining was performed, as we have previously reported. ${ }^{10}$ Briefly, paraffin sections were stained using rabbit anti-AT1/AT2 receptor polyclonal antibodies, and a rabbit polyclonal anti-ACE2 antibody (Santa Cruz Biotechnology), a rabbit monoclonal antibody against macrophages (CD68; EMD Millipore, Billerica, MA, USA), and a monoclonal antibody used against alpha smooth muscle actin ( $\alpha$-SMA; Sigma-Aldrich $\mathrm{Co}$ ). The slides were incubated with a biotinylated horse anti-rabbit IgG or an anti-mouse IgG (Vector Laboratories, Inc, Burlingame, CA, USA), stained using the ABC peroxidase kit or ABC-AR kit (alkaline phosphatase; Vector Laboratories, Inc), and substrated with a 3,3'-diaminobenzidine tetrahydrochloride or alkaline phosphatase substrate kit (Sigma-Aldrich Co). Quality of immunohistochemistry assay was controlled by either elimination of the primary antibody or incubation of the tissue with a non-immune IgG. The intensity in expression of AT1/AT2 receptors and ACE2, as well as accumulation of macrophages and $\alpha$-SMA-expressing myofibroblasts among groups, were compared using computer-assisted morphometry (ImageJ software). The final results were averaged from the eight randomized high-powered fields.

\section{Evaluation of collagen deposition and tissue fibrosis}

Collagen deposition and fibrosis formation in the myocardium were evaluated using Masson's trichrome staining. Briefly, the paraffin sections were deparaffinized, hydrated with distilled water, and stained with Masson's trichrome method. It is an accurate technique for evaluating collagen deposition within muscle as we have reported previously. ${ }^{10}$ The staining protocol produces collagen blue, nuclei black, and viable muscle fiber red. Eight randomized high-powered fields per tissue section were examined for positively stained deposition (ie, blue staining in the peri-vascular area and in the myocardium) using a digital image analyzer (Image J software).

\section{Statistical analysis}

All data were reported as the mean \pm standard error. A one-way analysis of variance (ANOVA) followed by the Student-Newman-Keuls post hoc test was used to analyze group differences in the intensity of AT1/AT2 receptors and ACE2, populations of macrophages and myofibroblasts, and the expression of TGF $\beta 1$, collagens, and phospho-Smads. 
Blood pressure data were analyzed by one-way repeated measures ANOVA followed by post hoc analysis with the Student-Newman-Keuls test for multiple comparisons at different time points using SigmaPlot (Systat Software Inc, San Jose, CA, USA). Statistical significance was set at a value of $P<0.05$.

\section{Results}

\section{Effect of treatment with dietary curcumin on Ang II infusion-induced change in arterial blood pressure}

There was no group difference detected in systolic and diastolic pressures at baseline before osmotic pump implantations. As shown in Figure 1, in the sham (normal) group, arterial blood pressure calculated as a mean arterial pressure (MAP) remained at normal levels throughout the 28-day time course. However, subcutaneous infusion of Ang II significantly increased MAP compared to the baseline values during the observational periods, wherein MAP rose from $83.4 \pm 6.4 \mathrm{mmHg}$ at baseline to $155.3 \pm 8.3 \mathrm{mmHg}$ on day 7 , and achieved its maximum value of $212.0 \pm 9.1 \mathrm{mmHg}$ on
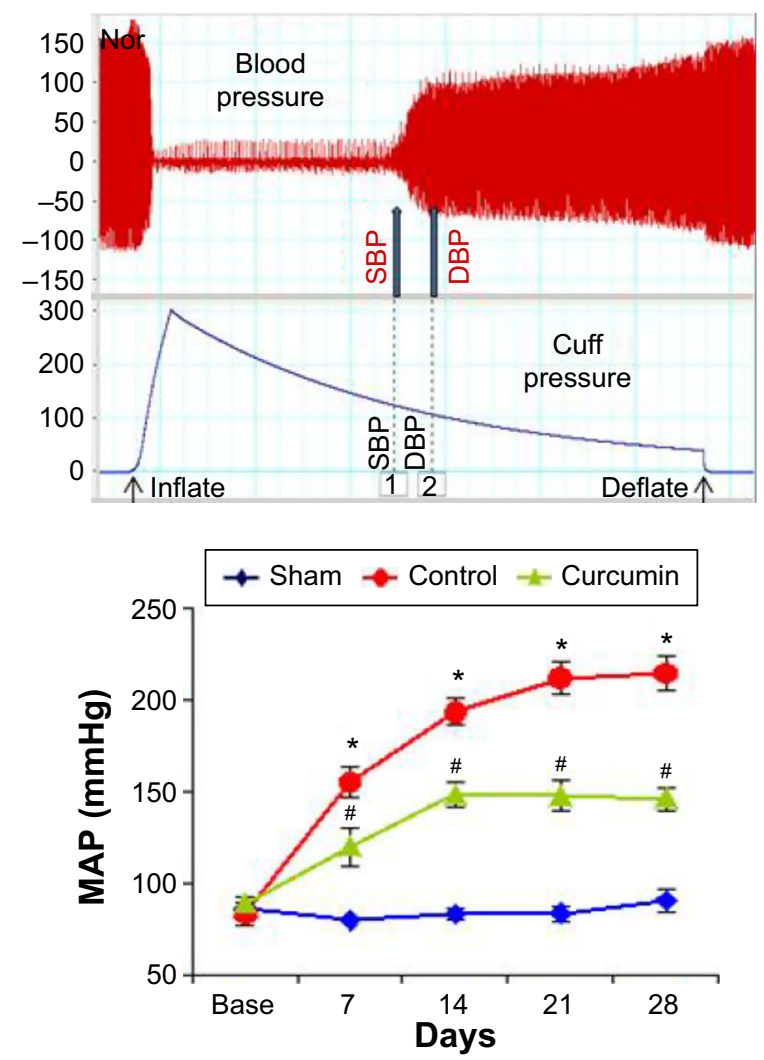

Figure I Representative tracing of pulse signals by inflating the tail cuff with simultaneous monitoring of the cuff pressure. The arrows indicate systolic blood pressure (SBP) and diastolic blood pressure (DBP). Difference in blood pressure among groups can be identified by a distance between cuff inflation from baseline (Base) and the beginning of SBP tracing. Treatment with dietary curcumin reduced blood pressure, as calculated from a mean arterial pressure (MAP) during Ang II infusion.

Notes: Values are mean \pm SEM; $n=6$ for each group. $* P<0.05$ Ang II infusion (Con) vs sham (Nor); ${ }^{*}<<0.05$ curcumin (Cur) vs Con.

Abbreviations: Ang II, angiotensin II; Con, control Ang II infusion treatment group; Nor, normal sham curcumin treatment group; Cur, curcumin plus Ang II treatment group; $n$, number of rats; SEM, standard error of the mean.

day 21 (all $P<0.05$ ). MAP remained at an elevated level throughout the experimental period in the control group. Treatment with dietary curcumin reduced MAP in comparison with the control animals. MAP was found to be significantly lower in the curcumin group (ie, Aug II and curcumin) when compared to the control group at each time point during Ang II infusion.

\section{Down-regulation of ATI receptor and up-regulation of AT2 receptor expression}

AT1 and AT2 receptor proteins in the myocardial proteomes were analyzed by Western blotting, and their expression was assessed with immunohistochemistry. As shown in Figure 2A, AT1 receptor expression was detected in the myocardium of the sham group. Ang II infusion caused a significant increase in protein level of the AT1 receptor at week 4 relative to the sham group. Consistent with Ang II-induced up-regulation of AT1 receptor protein level, expression of the AT1 receptor in the myocardium was also enhanced to a significant level in the control group, as revealed by immunohistochemical staining. As shown in Figure 2B, no positive
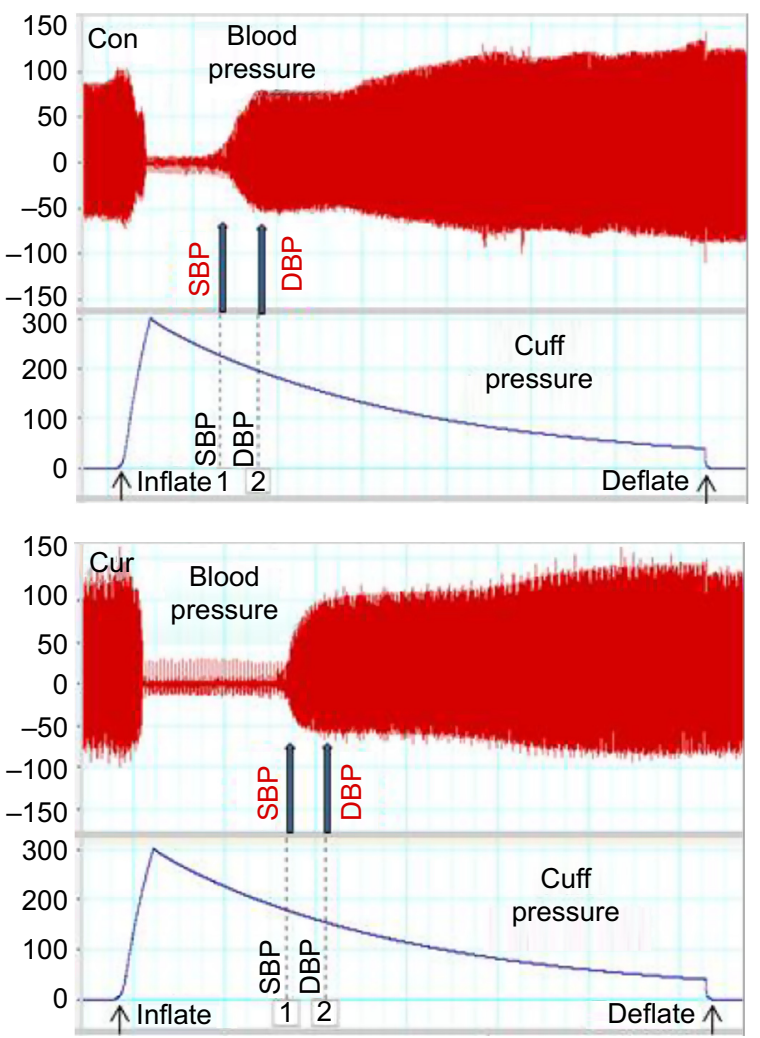
A

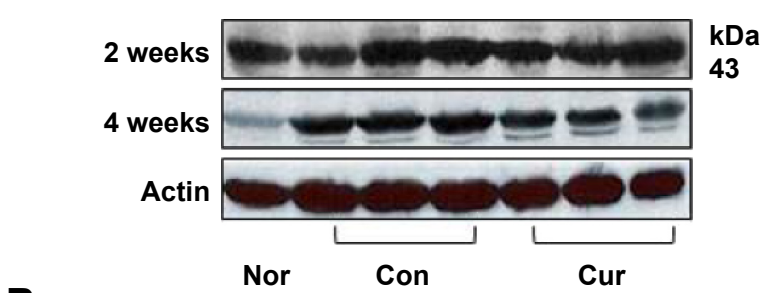

B
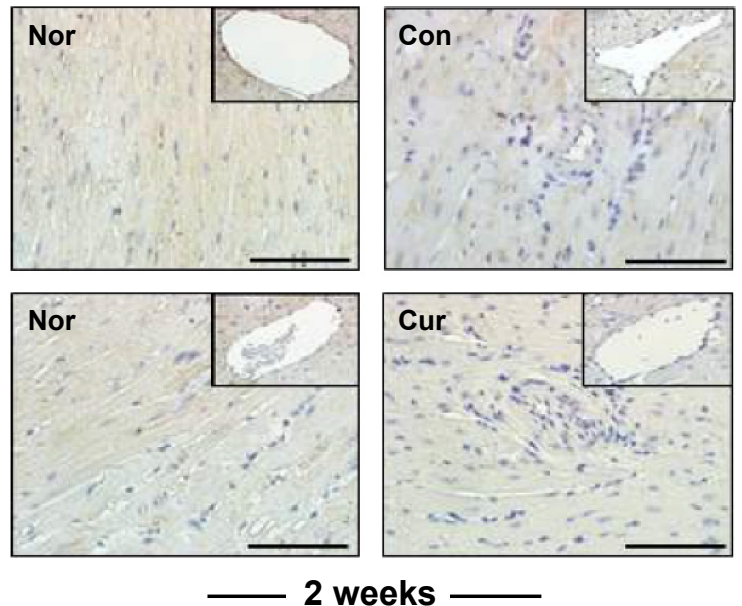
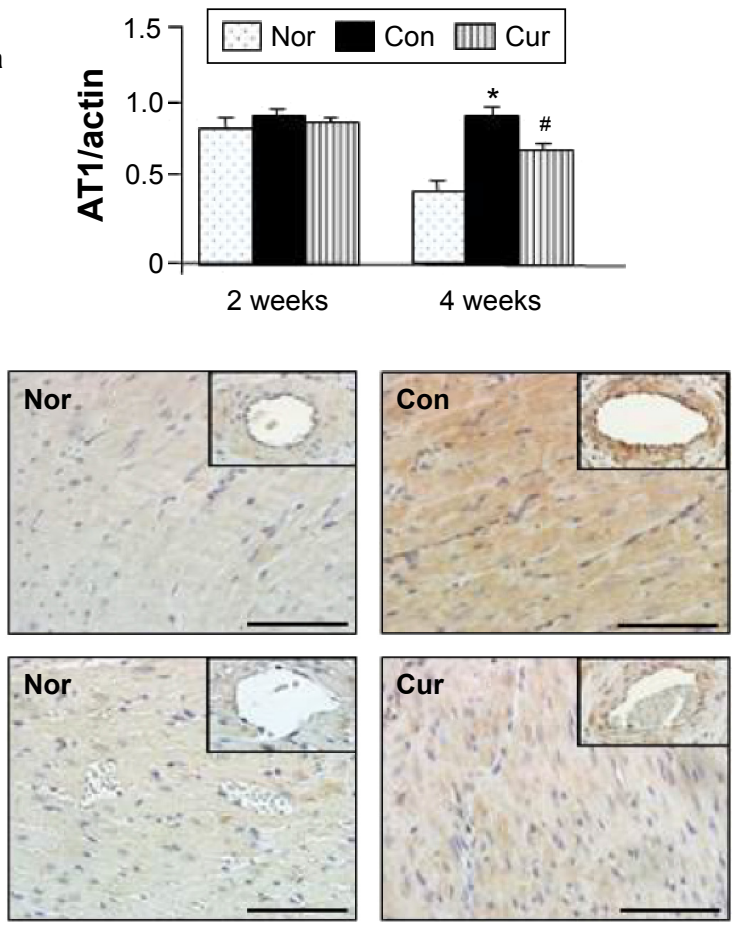

4 weeks

Figure 2 Detection of the ATI receptor during Ang II infusion.

Notes: These are representatives of tissue ATI receptor protein level by Western blotting $(\mathbf{A})$ and ATI receptor expression by immunohistochemical staining (original magnification $\times 400$; scale bars $=100 \mu \mathrm{m}$ ) in the myocardium $(\mathbf{B})$ and intracardiac vessels (Inset images in B), which were significantly attentuated by curcumin. All bands at week 2 and 4 were normalized by actin as a loading control. Values are mean $\pm S E M ; n=6$ for each group. ${ }^{*} P<0.05$ Ang II infusion (Con) vs sham (Nor); ${ }^{\sharp} P<0.05$ curcumin (Cur) vs Con.

Abbreviations: Ang II, angiotensin II; Con, control Ang II infusion treatment group; Nor, normal sham curcumin treatment group; Cur, curcumin plus Ang II treatment group; n, number of rats; SEM, standard error of the mean; ATI, Ang II type I receptor.

immunostaining for AT1 receptor in the myocardium was found during the course of the experiment in the sham group. However, the immunostaining intensity of the AT1 receptor was markedly enhanced in the peri-vascular area and myocardium at week 4 following Ang II infusion. Treatment with dietary curcumin over the period of the experiment abrogated the up-regulation of AT1 receptors. The expression of AT1 in the proteome in the intracardiac vessels and in the myocardium was significantly reduced when compared with control animals at week 4.

Analyses of tissue AT2 protein levels and expression of the AT2 receptor were also conducted using Western blotting and immunohistochemistry. As shown in Figure 3A, the AT2 receptor protein was constitutively presented and expressed in the peri-vascular area and myocardium in the sham group (Figure 3B). However, protein level and expression of the AT2 receptor in the control group were significantly reduced during 2 and 4 weeks of Ang II infusion, respectively, relative to those in the sham group. Along with an inhibition in expression of the AT1 receptor with dietary curcumin, the down-regulated AT2 receptor in the intracardiac vessels and myocardium was significantly increased when compared with the control. These results suggested that curcumin has dual effects on Ang II-modulated AT1 and AT2 receptors. This was further supported by an increased ratio of the AT2 receptor over the AT1 receptor after 4 weeks of Ang II infusion in the curcumin group $(1.2 \pm 0.02 \%)$ vs in the control $(0.7 \pm 0.03 \%, P<0.05)$.

\section{Inhibition of macrophages accumulation and myofibroblast proliferation}

Monocyte-derived macrophages are known to be involved in proliferation and differentiation of fibroblasts to myofibroblasts. ${ }^{10}$ In the present study, we selected immunohistochemical staining to identify accumulation of macrophages and proliferation of myofibroblasts. As shown in Figure 4, no macrophages were detected in the myocardium in the sham group. Ang II infusion caused a significant increase in the number of macrophages in the myocardium at week 2 . This accumulation was maintained at a constant level until week 4 . The proliferation of fibroblasts was identified as accumulation of the $\alpha$-SMA-expressing myofibroblasts. As shown in Figure 5A, a few of $\alpha$-SMA-expressing myofibroblasts were only present 
A

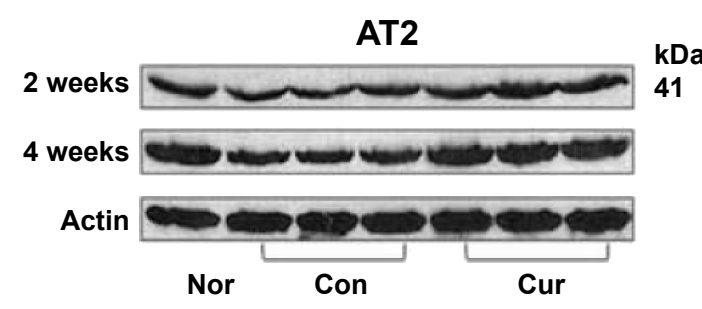

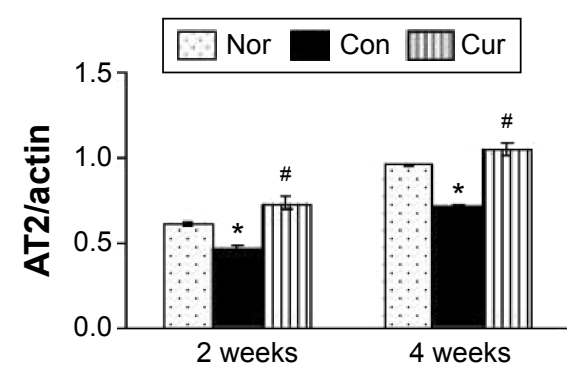

B
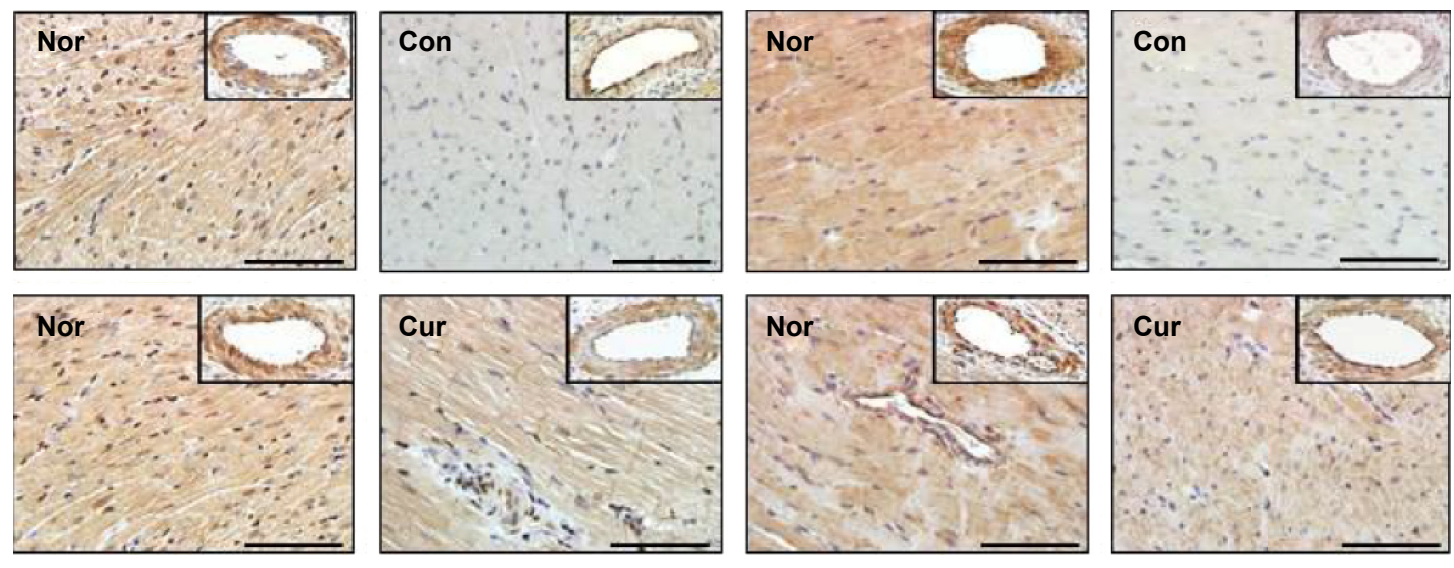

2 weeks

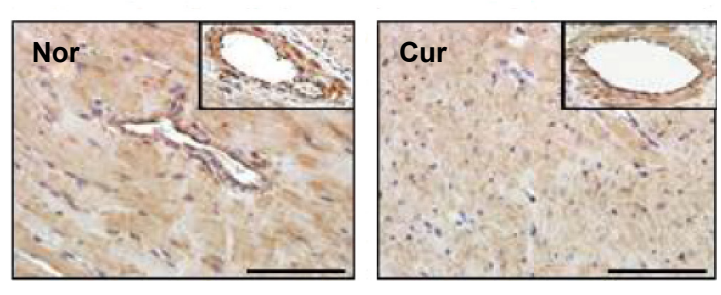

4 weeks

Figure 3 Detection of the AT2 receptor during Ang II infusion.

Notes: These images are representatives of tissue AT2 receptor protein levels by Western blotting (A) and AT2 receptor expression by immunohistochemical staining (original magnification $\times 400$; scale bars $=100 \mu \mathrm{m}$ ) in the myocardium $(\mathbf{B})$ and intracardiac vessels (Inset images in B), which were significantly attentuated by curcumin. All bands at week 2 and 4 were normalized by actin as a loading control. Values are mean \pm SEM; $n=6$ for each group. ${ }^{*} P<0.05$ Ang II infusion (Con) vs sham (Nor); ${ }^{P}<<0.05$ curcumin (Cur) vs Con.

Abbreviations: Ang II, angiotensin II; Con, control Ang II infusion treatment group; Nor, normal sham curcumin treatment group; Cur, curcumin plus Ang II treatment group; $n$, number of rats; SEM, standard error of the mean.

in the vascular smooth muscle, but not in the myocardium in the sham group. However, the number of $\alpha$-SMA-expressing myofibroblasts was significantly increased at weeks 2 and 4 during Ang II infusion relative to the sham group. The majority of myofibroblasts were aligned with the host myocardial fibers. Treatment with dietary curcumin over 4 weeks significantly reduced the numbers of accumulated macrophages and proliferated myofibroblasts at weeks 2 and 4 compared to their respective controls (Figures 4 and 5A).

\section{Attenuated expression of TGF $\beta$ I and phosphorylation of Smad2/3}

Activation of TGF $\beta 1 /$ Smads signaling pathways has been associated with proliferation of myofibroblasts. ${ }^{10}$ In the present study, we selected Western blotting assay to detect expression of TGF $\beta 1 /$ Smads. As shown in Figure 5B, TGF $\beta 1$ was significantly up-regulated with Ang II infusion at week 2 , and its expression was maintained at a high level at week 4, which was consistent with increased number of the proliferated myofibroblasts. Smad2, but not Smad3, was barely phosphorylated in the sham group (Figure 6).
Ang II infusion did not alter the level of phosphorylated Smad3, but significantly up-regulated phosphorylation of Smad2 at week 2. At week 4, both Smad2 and Smad3 were phosphorylated following Ang II infusion. Treatment with dietary curcumin during the 28 days of Ang II infusion comparatively abrogated expression of TGF $\beta 1$ and the phosphorylation of the $\mathrm{Smad} 2 / \mathrm{Smad} 3$ relative to their respective control.

\section{Reduced synthesis of collagen I and formation of fibrotic tissue}

Excessive collagen deposition has been linked to myocardial stiffness, and diastolic and systolic dysfunction. ${ }^{10}$ In the present study, we selected Western blotting assay to detect expression of fibrillar collagen type I, which is synthesized in the fibroblasts. ${ }^{3}$ As shown in Figure 7A, no collagen I was detected in the sham group during the 4 weeks of observation. Ang II infusion significantly increased the level of collagen I at week 2 and further enhanced its expression at week 4 . To confirm the results demonstrated by Western blotting assay, we selected Masson's trichrome staining to locate 

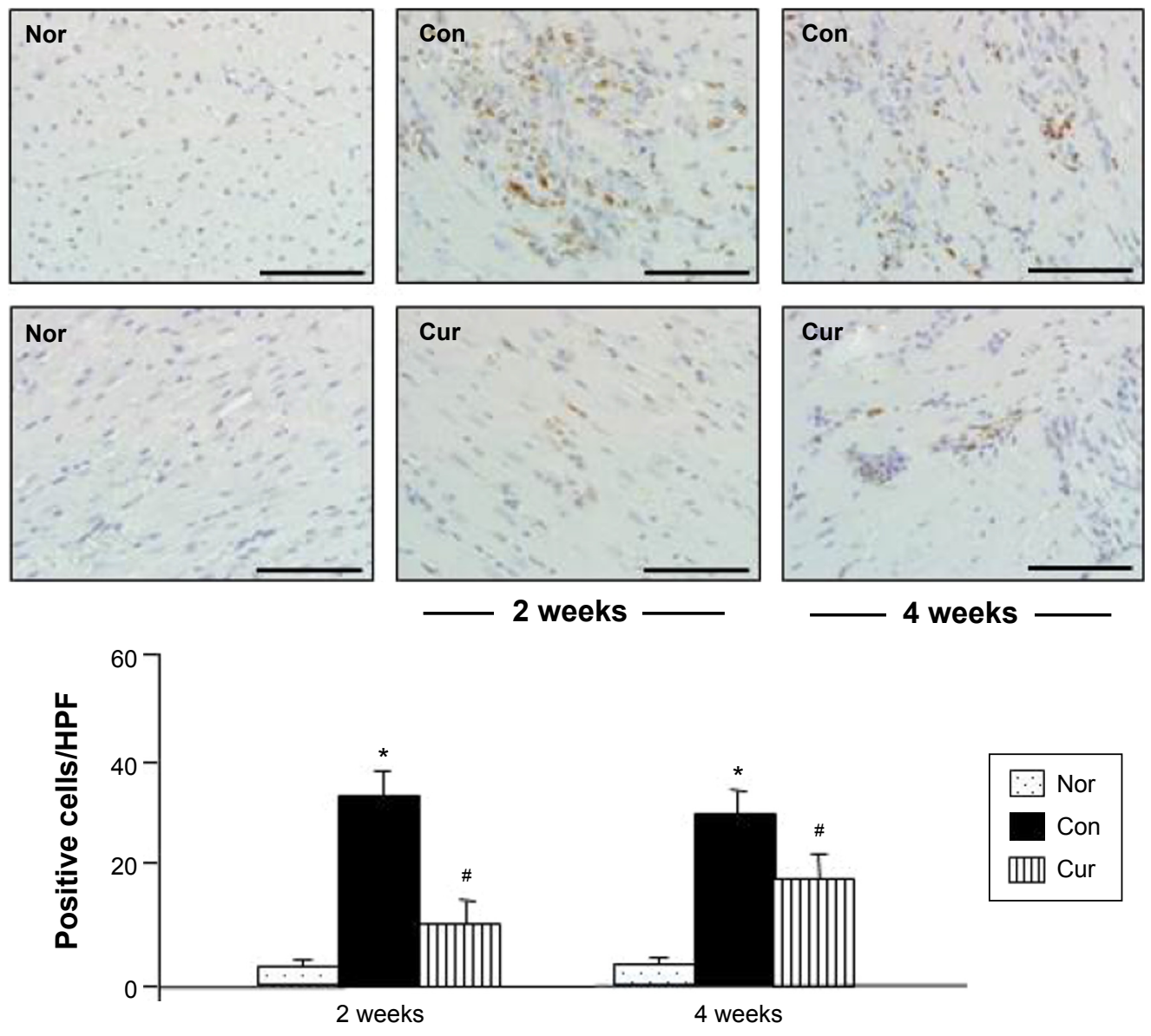

Figure 4 Macrophage accumulation during Ang II infusion. Accumulation of macrophages was detected using immunohistochemical staining. Ang II caused a significant increase in the number of positively stained macrophages during 2 and 4 weeks of Ang II infusion, which was significantly inhibited by curcumin.

Notes: Analysis was calculated by the numbers in each high-powered field (HPF; magnification $\times 400$; scale bars $=100 \mu \mathrm{m}$ ). Values are mean $\pm \mathrm{SEM}$; $\mathrm{n}=6$ for each group. $* P<0.05$ Ang II infusion (Con) vs sham (Nor); ${ }^{P} P<0.05$ curcumin (Cur) vs Con.

Abbreviations: Ang II, angiotensin II; Con, control Ang II infusion treatment group; Nor, normal sham curcumin treatment group; Cur, curcumin plus Ang II treatment group; n, number of rats; SEM, standard error of the mean; HPF, high-powered field.

fibrotic tissue in the myocardium. As shown in Figure 7B, collagen was only detected in the intracardiac vessels, but not in the myocardium, at the end of sham group observation. However, consistent with increased collagen I expression, deposition of fibrotic tissue within the peri-vascular region and myocardium was markedly enhanced at week 4 in the control group. Treatment with dietary curcumin over 4 weeks of Ang II infusion significantly reduced the level of the collagen I and the extent of the fibrosis in the intracardiac vessels and myocardium, as evidenced by more organized and circumscribed fibers.

\section{Augmented expression of ACE2 in the myocardium}

Proteomic and cellular expression of ACE2 was examined using Western blotting and immunohistochemistry, respectively. As shown in Figure 8A, ACE2 was constitutively expressed in the sham group and hypertensive rats in the control group. It was clearly shown that the presence of hypertension did not significantly alter the expression of ACE2 in the proteome relative to the sham control at week 2. Serial sections of immunohistochemistry in the sham and control animals also showed a similar pattern in expression of ACE2 in the myocardium (Figure 8B). However, at week 4, Ang II infusion significantly reduced ACE2's expression, which was reversed with 4 weeks of dietary curcumin treatment (Figure 8).

\section{Discussion}

Myocardial fibrosis-induced diastolic dysfunction is one of the most common cardiac abnormalities in heart failure patients. Ang II converted from Ang I by ACE is a peptide hormone that increases blood pressure to create its systemic hemodynamic effect by constricting blood vessels. Ang II also elicits myocardial fibrosis directly through fibroblastactivated TGF $\beta 1 /$ Smads signaling pathways. Our current 

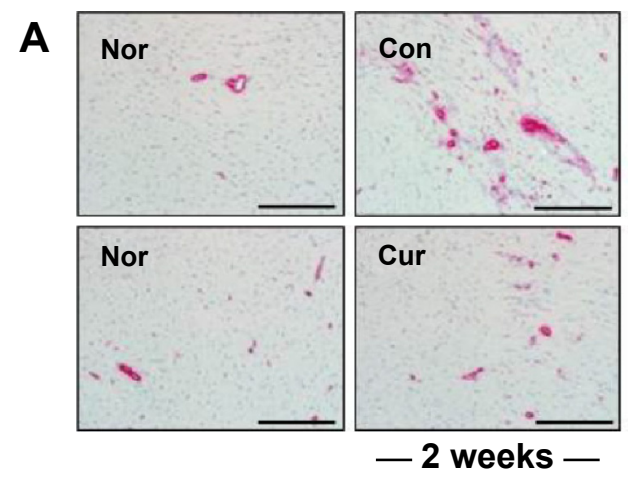

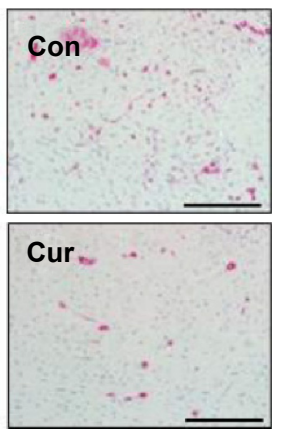

-4 weeks -

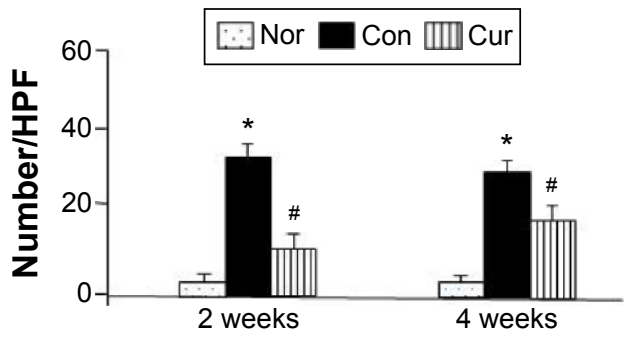

B

TGF $\beta 1$

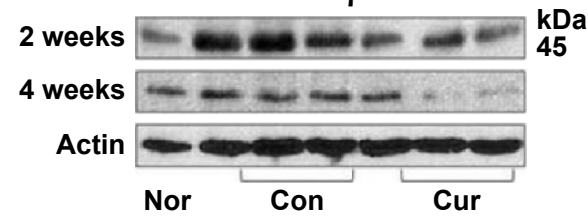

kDa
45

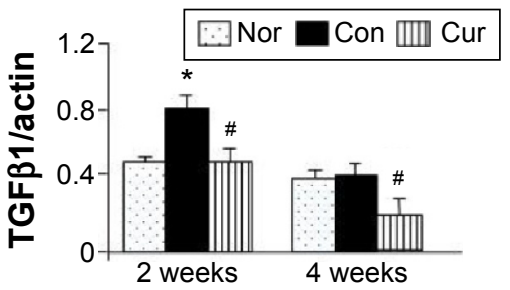

Figure 5 Proliferation of fibroblasts and expression of TGF $\beta$ I during Ang II infusion. Myofibroblast proliferation was detected using immunohistochemical staining, (A) and TGF $\beta$ I was analyzed using Western blotting (B). Ang II caused a significant increase in the number of proliferated fibroblasts, shown as $\alpha$-SMA-expressing myofibroblasts and up-regulation of TGF $\beta$ I at weeks 2 and 4 , which was significantly inhibited by curcumin.

Notes: Analysis was calculated by the numbers in each high-powered field (HPF; magnification $\times 400$; scale bars $=100 \mu \mathrm{m}$ ). Values are mean \pm SEM; $n=6$ for each group. $* P<0.05$ Ang II infusion (Con) vs sham (Nor); ${ }^{*} P<0.05$ curcumin (Cur) vs Con.

Abbreviations: TGF $\beta$ I, transforming growth factor beta I; Ang II, angiotensin II; $\alpha$-SMA, alpha smooth muscle actin; Con, control Ang II infusion treatment group; Nor, normal sham curcumin treatment group; Cur, curcumin plus Ang II treatment group; n, number of rats; SEM, standard error of the mean; HPF, high-powered field.

study revealed a novel paradigm for explaining the protective mechanisms of action by curcumin. We found that, during Ang II infusion, dietary administration of curcumin decreases protein level of the AT1 receptor and enhances expression of the AT2 receptor/ACE2. In accordance with these findings, the accumulation of macrophages/myofibroblasts and the formation of fibrotic tissue were attenuated. These results were consistent with previous reports showing antifibrotic effects of curcumin on other organ systems such as lung, ${ }^{11}$ liver, ${ }^{12}$ and kidney. ${ }^{13}$ Furthermore, curcumin, when given
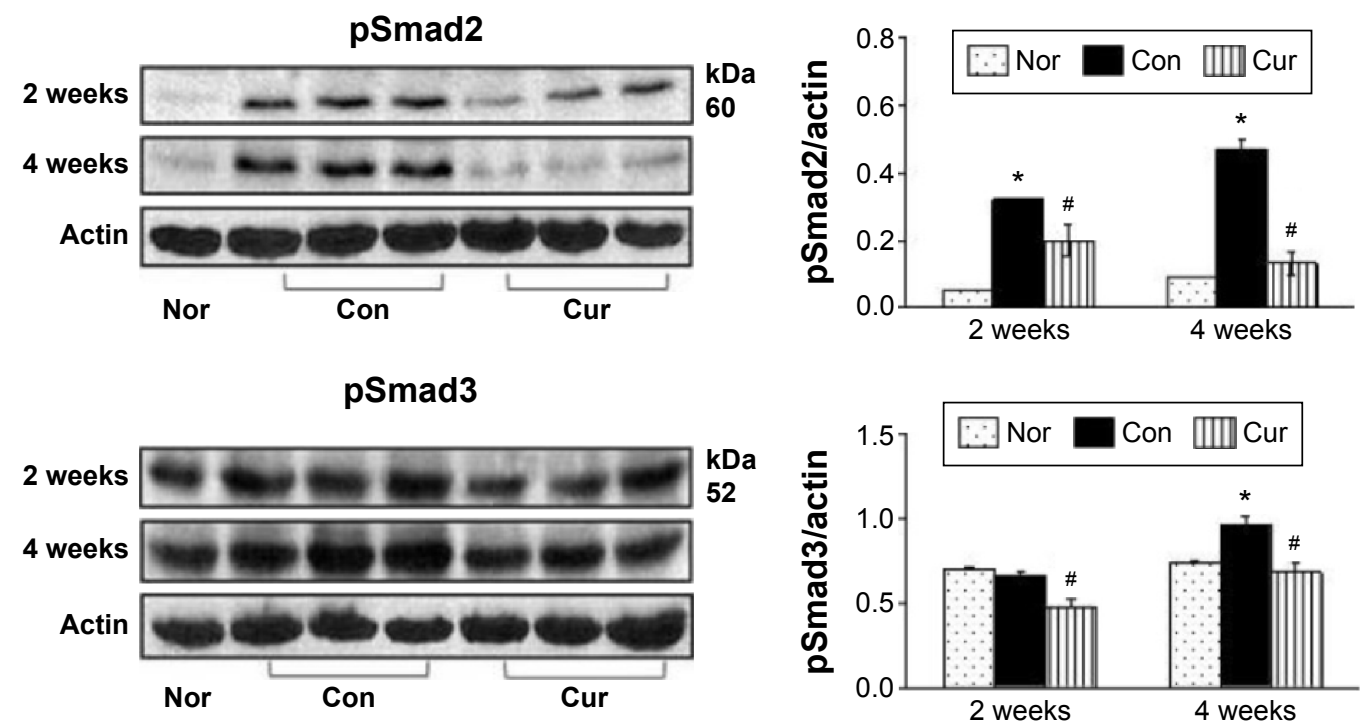

Figure 6 Phosphorylation of Smad2/3 during Ang II infusion. Ang II caused a significant increase in phospho-Smad2/3 levels at week 4, as normalized by actin for each band, which were significantly inhibited by curcumin.

Notes: Values are mean \pm SEM; $n=6$ for each group. $* P<0.05$ Ang II infusion (Con) vs sham (Nor); ${ }^{p}<0.05$ curcumin (Cur) vs Con.

Abbreviations: pSmad, phosphorylated Smad; Ang II, angiotensin II; Con, control Ang II infusion treatment group; Nor, normal sham curcumin treatment group; Cur, curcumin plus Ang II treatment group; $n$, number of rats; SEM, standard error of the mean. 
A
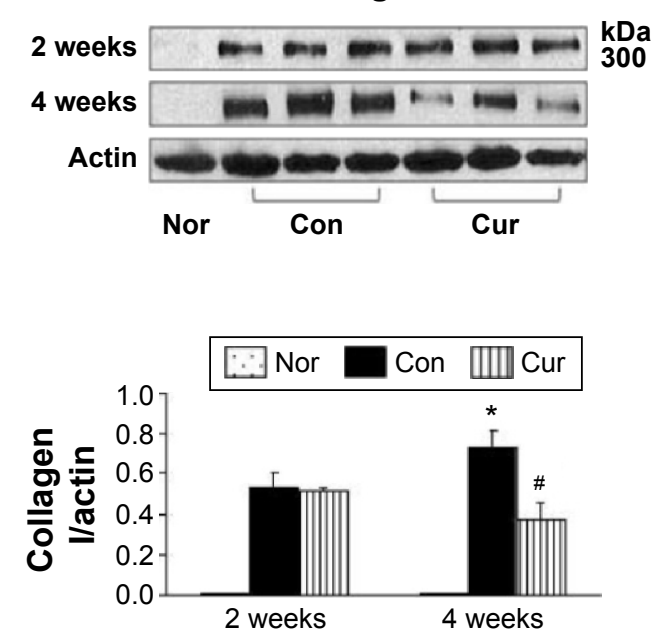
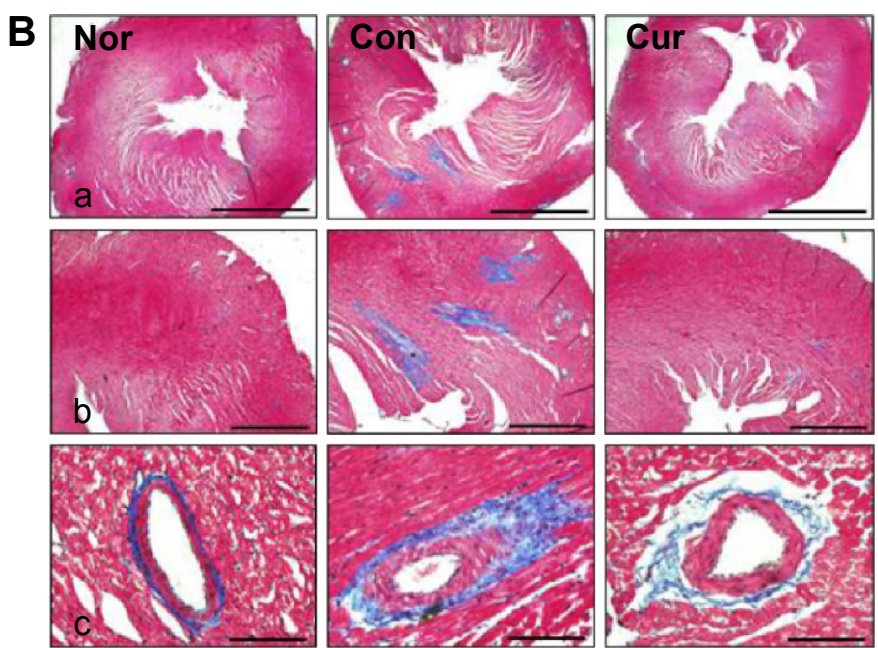

4 weeks

Figure 7 Expression of collagen type I and fibrotic tissue formation during Ang II infusion.

Notes: Ang II increased collagen type I levels at week 4, as normalized by actin for each band (A). Representative photomicrographs of fibrosis were taken from the tissue sections stained with Masson's trichrome staining (B). Ang II markedly enhanced collagen deposition in the intracardiac vessels and in the intermyocardium. Treatment with curcumin reduced the collagen type I level and the scope of fibrotic tissue at week 4. (Ba) Magnification $\times 200$; scale bars $=200 \mu \mathrm{m}$; $(\mathbf{B b})$ and $(\mathbf{B c})$, magnification $\times 400$; scale bars $=100 \mu \mathrm{m}$ at week 4. Values are mean \pm SEM; $n=6$ for each group. $* P<0.05$ Ang II infusion (Con) vs sham (Nor); $\#<0.05$ curcumin (Cur) vs Con.

Abbreviations: Ang II, angiotensin II; Con, control Ang II infusion treatment group; Nor, normal sham curcumin treatment group; Cur, curcumin plus Ang II treatment group; $n$, number of rats; SEM, standard error of the mean.

A

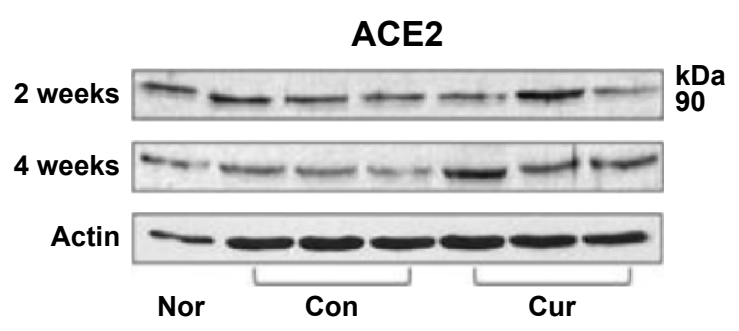

B
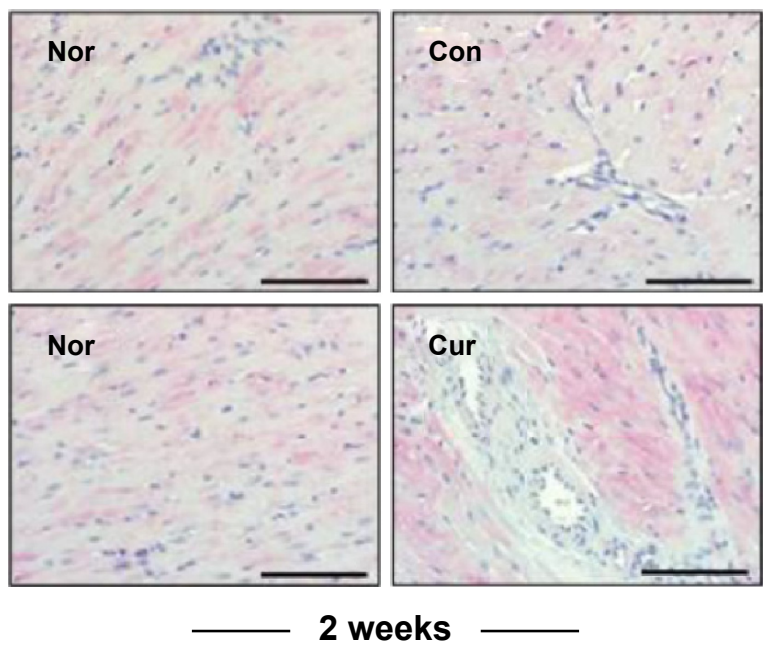
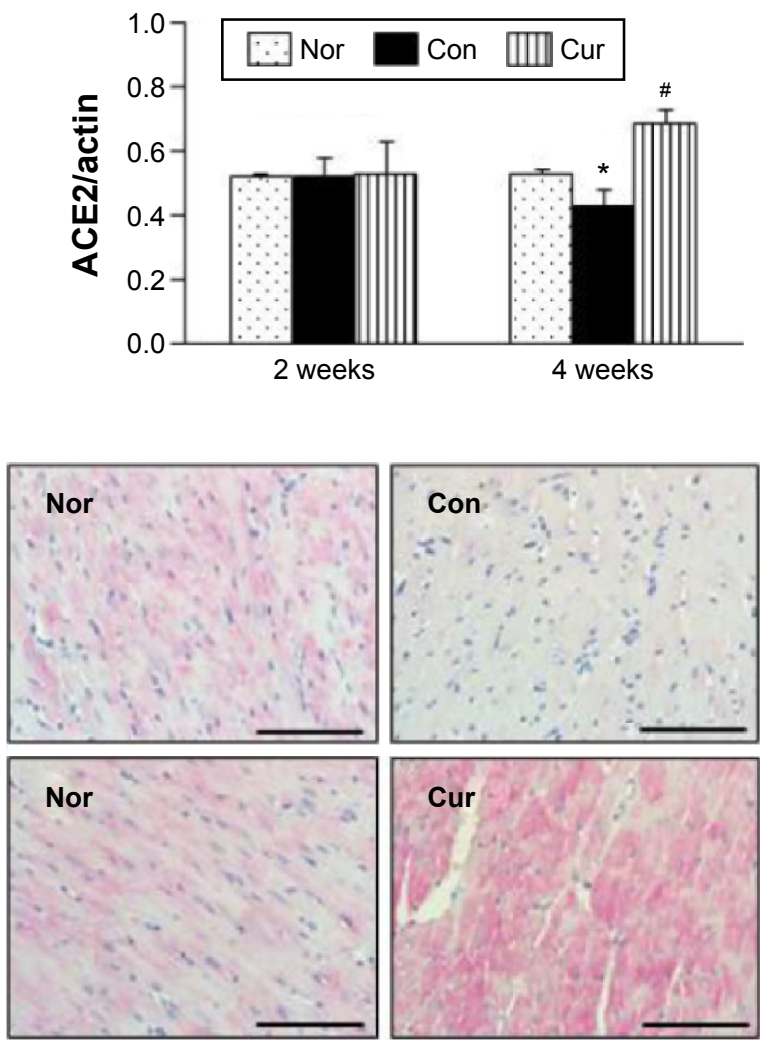

4 weeks

Figure 8 Expression of ACE2 during Ang II infusion.

Notes: Ang II significantly reduced ACE2 levels at week 4, as normalized by actin for each band, using Western blotting (A) and identified by immunohistochemical staining

(B), which was reversed by treatment with curcumin. Images were taken in a high-powered field (HPF; magnification $\times 400 ; s c a l e ~ b a r s=100 \mu m$ ). Values are mean \pm SEM; $n=6$ for each group. ${ }^{*} P<0.05$ Ang II infusion (Con) vs sham (Nor); ${ }^{P}<0.05$ curcumin (Cur) vs Con.

Abbreviations: ACE2, angiotensin-converting enzyme 2; Ang II, angiotensin II; Con, control Ang II infusion treatment group; Nor, normal sham curcumin treatment group; Cur, curcumin plus Ang II treatment group; n, number of rats; SEM, standard error of the mean; HPF, high-powered field. 
alone in the sham group in the current study, had no effect on blood pressure, but significantly reduced blood pressure in the presence of Ang II, suggesting that curcumin is capable of lowering blood pressure, potentially through its direct dilatory effect on blood vessels, by modulating Ang II receptors.

Ang II mediates the majority of its deleterious effects via the AT1 receptor, whereas activation of the AT2 receptor counter-regulates actions of the AT1 receptor. ${ }^{14,15}$ In the current study, we selected a rat model of Ang II infusion, wherein we provided a constant supply of Ang II in tissue with an osmotic pump. Augmented plasma and tissue levels of Ang II with an osmotic pump infusion have been accomplished previously. ${ }^{16}$ The model used in the present study allowed us to demonstrate direct effect of Ang II on myocardial fibrosis, without considering the role of ACE in Ang II production. Obviously, there was a reciprocal expression between the AT1 receptor and the AT2 receptor in response to Ang II infusion in the present study, with up-regulated AT1 receptors and down-regulated AT2 receptors evident. They were inversely correlated, as demonstrated by the densitometry results from Western blotting analysis. Because Ang II binds to the AT1 receptor and the AT2 receptor subtypes with similar affinities, the deleterious effects of Ang II are highly dependent upon the relative expression levels of both receptors. ${ }^{15}$ Histological analysis by immunohistochemistry confirmed that positive immunoreactivity of the AT1 and AT2 receptors exists in both the peri-vascular area and the intermyocardium. Along with down-regulated expression and localization of the AT1 receptor by curcumin, expression of the AT2 receptor over 4 weeks of Ang II infusion was significantly up-regulated, further suggesting that there is cross-talk between these two receptors. These results were consistent with a previous study showing that down-regulation of the AT1 receptor up-regulates AT2 receptor expression. ${ }^{15}$ To more fully understand and to clinically utilize this regulatory circuit, future studies that evaluate the effects of curcumin on Ang II-targeted binding kinetics on AT1/AT2 receptors are warranted.

It has been reported previously that histological features of Ang II-induced tissue remodeling to adapt to pressure overload include myocyte hypertrophy and myocardial fibrosis, which develop from the peri-vascular space into the intermuscular interstitium. ${ }^{3}$ At 4 weeks of Ang II infusion, we found that collagen deposition, as revealed by Masson's trichrome staining, was detected in both peri-vascular and intermuscular regions, suggesting a development of reactive fibrosis. In response to Ang II stimulation, myocardial fibrosis can be induced through pressure-dependent or -independent mechanisms. These mechanisms include many interacting factors such as mechanical stretch of myocytes, coronary perfusion with high blood pressure, and inflammation-triggered accumulation of macrophages/myofibroblasts. Accordingly, attenuation of blood pressure or inhibition of inflammation have been shown to reduce reactive myocardial fibrosis. ${ }^{10,16,17}$ In the present study, curcumin at the dose of $150 \mathrm{mg} / \mathrm{kg} /$ day significantly reduced blood pressure. This action was potentially mediated by attenuated blood vessel contraction through modulation of AT1/AT2 receptor expression. It is noteworthy that treatment with dietary curcumin inhibits oxidative stress and inflammation, as we have reported previously. ${ }^{10}$ Moreover, curcumin attenuates fibroblast proliferation and TGF $\beta 1 /$ Smads expression, as noted in the present study; this action may concomitantly attenuate reactive myocardial fibrosis via a pressure-dependent or -independent pathway.

Previous studies have shown that ACE2 is presented in the vascular endothelium, smooth muscle cells of coronary vessels, and cardiomyocytes. ${ }^{5,6}$ In the present study, ACE2 expression was examined from transmural tissue blocks using Western blotting and immunohistochemical staining. Although we could not differentiate the locations responsible for ACE2 expression, we found that Ang II infusion over 4 weeks caused significant suppression of ACE2 expression, as shown in Figure 8. Consistent with action of AT1 receptor antagonism by dietary curcumin, Ang II-reduced ACE2 expression was also abolished with curcumin, indicating that Ang II signaling pathway via AT1 receptor activation may, in part, be responsible for down-regulation of ACE2 expression. These results were consistent with a previous study showing that ACE2 expression is up-regulated using the Ang II AT1 receptor antagonist. ${ }^{18}$ Given the key roles of ACE2 in the degradation of Ang II into the vasodilator, and its antihypertrophic and anti-inflammation actions on the heart, ${ }^{6,18}$ we predicted that maintaining myocardial ACE2 level by curcumin would negate the detrimental effects of Ang II. These results were also consistent with previous reports, wherein similar pharmacological interventions aimed at modulating ACE2 and the AT2 receptor reduced cardiac fibrosis. ${ }^{19-21}$ Taken together, the actions that enhance ACE2 expression and that alter AT1/AT2 receptors may further enhance inhibitory effects of curcumin on myocardial fibrosis.

Myocardial fibrosis during Ang II infusion is characterized by abundant collagen deposition without significant formation of myocardial necrosis and scar tissue. Induction of myocardial fibrosis by Ang II has been associated with macrophage-triggered differentiation of fibroblasts 
into myofibroblasts through TGF $\beta 1 /$ Smads-dependent signaling. ${ }^{22,23}$ Through activating the AT1 receptor on macrophages, Ang II stimulates macrophages to release soluble substances, such as TGF $\beta 1$, responsible for induced collagens via Smad-mediated signaling pathways by proliferated myofibroblasts. In correspondence with a reduction in accumulation of macrophages by curcumin, we found that proliferation of fibroblasts to myofibroblasts was also inhibited, indicating that macrophages function as a master regulator of myofibroblast activation. Stimulated myofibroblasts caused phosphorylation of $\operatorname{Smad} 2 / 3$ to form a heterotrimeric complex with Smad4, and subsequently were bound to TGF $\beta 1$-targeted collagen genes in the nucleus for production of collagens. During 4 weeks of Ang II infusion, we found that Smad2 and Smad3 were phosphorylated and that tissue-level expression of collagen type I was increased, consistent with enhanced TGF $\beta 1$ expression. Treatment with curcumin inhibited the up-regulation of both TGF $\beta 1$ and $\mathrm{Smad} 2 / 3$, and therefore, reduced synthesis of collagen I, as evidenced by less formation of collagen-rich fibrotic tissue in the peri-vascular space and intermyocardium. In this regard, data from our lab and others have previously shown that treatment with curcumin on a daily basis balances the synthesis and degradation of existing collagen matrix through inhibiting matrix metalloproteinases and myofibroblast/TG $\beta 1 /$ Smads signaling pathways after myocardial infarction. ${ }^{10-13}$ Extensive preclinical studies have established a solid basis for the application of curcumin against a wide range of cardiovascular diseases. ${ }^{24-26}$ Clinical trials have also addressed the pharmacokinetics, safety, efficacy, and therapeutic potential of curcumin in normal volunteers and in treating various human diseases including cancers, inflammation, diabetes, arterial stiffness, etc, in patients. ${ }^{26}$ Our current data provide further evidence that dietary curcumin could potentially be selected as a safe and effective treatment in fibrosis-derived heart failure.

\section{Study limitations}

The current study has several limitations. First, although it has been shown that myocardial ACE2 level in response to Ang II stimulation is associated with an up-regulated AT1 receptor via inflammatory response enhancement, ${ }^{5}$ our current study provides evidence showing an inhibition of the AT1 receptor and an augmentation of the ACE2 expression by curcumin. In view of these findings, it is the question of whether down-regulation of the AT1 receptor or up-regulation of the AT2 receptor by curcumin is due to decreased synthesis or increased degradation requires further exploration; methodology for future exploration may include measuring functional plasma membrane receptor numbers and Ang II receptor transcription levels. Second, myocardial fibrosis can be induced through a blood pressure-dependent or -independent manner, and our results showed that curcumin reduces blood pressure and inhibits fibrotic pathways. However, we do not know whether they are correlated. Using a model of transverse aorta constriction might allow us to differentiate these effects of curcumin on myocardial fibrosis. Third, it has previously been reported that encapsulated curcumin in different materials could increase its water solubility, plasma half-life, and tissue distribution. ${ }^{27,28}$ However, we do not know whether modified curcumin could exert a more potent effect on Ang II-induced myocardial fibrosis. Finally, we do not know whether inhibition of Ang II-induced cardiac fibrosis by curcumin could improve cardiac diastolic or systolic cardiac function. Therefore, more functional studies are necessary to demonstrate the final cardiac outcomes effected by curcumin, and these studies may require a complete hemodynamic assessment.

\section{Conclusion}

In the present study, we found that dietary treatment with curcumin reduced expression of the AT1 receptor and enhanced expression of both the AT2 receptor and ACE2. These results suggest that curcumin modulates not only Ang II/AT1/AT2 receptor-dependent signaling pathways, but also activates an ACE2-mediated mechanism that modulates myocardial fibrosis. Given the fact that 1) it is still questionable whether cardiac function could be improved additively by treatment with both ACE inhibitor and AT1 receptor antagonists in patients, ${ }^{29,30}$ and 2) cardiac function is further improved when animals are treated with curcumin and enalapril, ${ }^{24}$ therefore curcumin might be selected to combine with other existing therapeutic agents such as ACE inhibitor, AT1 antagonist, or beta receptor blocker to improve the final outcome in patients with myocardial fibrosis-derived heart failure.

\section{Acknowledgments}

This study was supported in part by grants from the Mercer University School of Medicine, the Medcen Community Health Foundation, Savannah, Georgia, and the National Natural Science Foundation of China (grants 81170145, 81470436).

\section{Disclosure}

The authors report no conflicts of interest in this work. 


\section{References}

1. McMurray JJ, Pfeffer MA. Heart failure. Lancet. 2005;365(9474): 1877-1889.

2. Liehn EA, Postea O, Curaj A, Marx N. Repair after myocardial infarction between fantasy and reality: the role of chemokines. $J$ Am Coll Cardiol. 2011;58(23):2357-2362.

3. Weber KT. Fibrosis in hypertensive heart disease: focus on cardiac fibroblasts. J Hypertens. 2004;22(1):47-60.

4. Crowley MJ, Powers BJ, Myers ER, McBroom AJ, Sanders GD. Angiotensin-converting enzyme inhibitors and angiotensin II receptor blockers for treatment of ischemic heart disease: future research needs prioritization. Am Heart J. 2012;16:(5):777-782.

5. Keidar S, Kaplan M, Gamliel-Lazarovich A. ACE2 of the heart: from angiotensin I to angiotensin (1-7). Cardiovascular Res. 2007;73(3): 463-469.

6. Yamamoto K, Ohishi M, Katsuya T, et al. Deletion of angiotensinconverting enzyme 2 accelerates pressure overload-induced cardiac dysfunction by increasing local angiotensin II. Hypertension. 2006;47(4): 718-726.

7. Yancy CW, Jessup M, Bozkurt B, et al; American College of Cardiology Foundation; American Heart Association Task Force on Practice Guidelines. 2013 ACCF/AHA guideline for the management of heart failure: a report of the American College of Cardiology Foundation/ American Heart Association Task Force on Practice Guidelines. $J$ Am Coll Cardiol. 2013;62(16):e147-e239.

8. Zhang Y, Jiang X, Peng K, et al. Discovery and evaluation of novel anti-inflammatory derivatives of natural bioactive curcumin. Drug Des Devel Ther. 2014;8:2161-2171.

9. Bhaskar Rao A, Prasad E, Deepthi SS, et al. Wound healing: a new perspective on glucosylated tetrahydrocurcumin. Drug Des Devel Ther. 2015;9:3579-3588.

10. Wang NP, Wang ZF, Tootle S, Philip T, Zhao ZQ. Curcumin promotes cardiac repair and ameliorates cardiac dysfunction following myocardial infarction. Br J Pharmacol. 2012;167(7):1550-1562.

11. Punithavathi $D$, Venkatesan N, Babu M. Protective effects of curcumin against amiodarone-induced pulmonary fibrosis in rats. Br J Pharmacol. 2003;139(7):1342-1350.

12. Xu J, Fu Y, Chen A. Activation of peroxisome proliferator-activated activated receptor-gamma contributes to the inhibitory effects of curcumin on rat hepatic stellate cell growth. Am J Physiol Gastrointest Liver Physiol. 2003;285(1):G20-G30.

13. Zhou XG, Zhang J, Xu C, Wang W. Curcumin ameliorates renal fibrosis by inhibiting local fibroblast proliferation and extracellular matrix deposition. J Pharmacol Sci. 2014;126(4):344-350.

14. Jones ES, Vinh A, McCarthy CA, Gaspari TA, Widdop RE. AT2 receptors: functional relevance in cardiovascular disease. Pharmacol Ther. 2008;120(3):292-316.

15. Namsolleck P, Recarti C, Foulquier S, Steckelings UM, Unger T. AT(2) receptor and tissue injury: therapeutic implications. Curr Hypertens Res. 2014;16(2):416
16. Zou LX, Hymel A, Imig JD, Navar LG. Renal accumulation of circulating angiotensin II in angiotensin II-infused rats. Hypertension. 1996;27(3 Pt 2):658-662.

17. Qi G, Jia L, Li Y, et al. Angiotensin II infusion-induced inflammation, monocytic fibroblast precursor infiltration, and cardiac fibrosis are pressure dependent. Cardiovasc Toxicol. 2011;11(2):157-167.

18. Koka V, Huang XR, Chung AC, Wang W, Truong LD, Lan HY. Angiotensin II up-regulates angiotensin I-converting enzyme (ACE), but down-regulates ACE2 via the AT1-ERK/p38 MAP kinase pathway. Am J Pathol. 2008;172(5):1174-1183.

19. Tokuda K, Kai H, Kuwahara F, et al. Pressure-independent effects of angiotensin II on hypertensive myocardial fibrosis. Hypertension. 2004;43(2):499-503.

20. Flores-Munoz M, Work LM, Douglas K, et al. Angiotensin-(1-9) attenuates cardiac fibrosis in the stroke-prone spontaneously hypertensive rat via the angiotensin type 2 receptor. Hypertension. 2012;59(2):300-307.

21. Patel VB, Clarke N, Wang Z, et al. Angiotensin II induced proteolytic cleavage of myocardial ACE2 is mediated by TACE/ADAM-17: a positive feedback mechanism in the RAS. J Mol Cell Cardiol. 2014; 66:167-176.

22. Kurdi M, Booz GW. New take on the role of angiotensin II in cardiac hypertrophy and fibrosis. Hypertension. 2011;57(6):1034-1038.

23. Hao JM, Wang B, Jones SC, Jassal DS, Dixon IM. Interaction between angiotensin II and Smad proteins in fibroblasts in failing heart and in vitro. Am J Physiol Heart Circ Physiol. 2000;279(6):H3020-H3030.

24. Sunagawa Y, Morimoto T, Wada H, et al. A natural p300-specific histone acetyltransferase inhibitor, curcumin, in addition to angiotensinconverting enzyme inhibitor, exerts beneficial effects on the left ventricular systolic function after myocardial infarction in rats. Circ $J$. 2011;75(9):2151-2159.

25. Meng Z, Yu XH, Chen J, Li L, Li S. Curcumin attenuates cardiac fibrosis in spontaneously hypertensive rats through PPAR- $\gamma$ activation. Acta Pharmacol Sin. 2014;35(10):1247-1256.

26. Gupta SC, Patchva S, Aggarwal BB. Therapeutic roles of curcumin: lessons learned from clinical trials. AAPS J. 2013;15(1):195-218.

27. Kim TH, Jiang HH, Youn YS, et al. Preparation of characterization of water-soluble albumin-bound curcumin nanoparticles with improved antitumor activity. Int J Pharm. 2011;403(1-2):285-291.

28. Bukhari SN, Jantan I, Unsal Tan O, Sher M, Naeem-Ul-Hassan M, Qin HL. Biological activity and molecular docking studies of curcuminrelated $\alpha, \beta$-unsaturated carbonyl-based synthetic compounds as anticancer agents and mushroom tyrosinase inhibitors. J Agric Food Chem. 2014;62(24):5538-5547.

29. Schindler C. ACE-inhibitor, AT1-receptor-antagonist, or both? A clinical pharmacologist's perspective after publication of the results of ONTARGET. The Adv Cardiovasc Dis. 2008;2(4):233-248.

30. Azizi M, Ménard J. Combined blockade of the renin-angiotensin system with angiotensin-converting enzyme inhibitors and angiotensin II type 1 receptor antagonists. Circulation. 2004;109(21):2492-2499.
Drug Design, Development and Therapy

\section{Publish your work in this journal}

Drug Design, Development and Therapy is an international, peerreviewed open-access journal that spans the spectrum of drug design and development through to clinical applications. Clinical outcomes, patient safety, and programs for the development and effective, safe, and sustained use of medicines are a feature of the journal, which
Dovepress

has also been accepted for indexing on PubMed Central. The manuscript management system is completely online and includes a very quick and fair peer-review system, which is all easy to use. Visit http://www.dovepress.com/testimonials.php to read real quotes from published authors. 